\title{
"Dry Arthroscopy" is a Valuable Tool in the Excisional Curettage of Chondroblastoma: A Case Series
}

\author{
Alec S Kellish ${ }^{1}$, Mahir Qureshi ${ }^{1}$, Andrew Mostello ${ }^{2}$, Tae Won Kim ${ }^{3}$, Christina J Gutowski ${ }^{3}$
}

Learning Point of the Article:

"Dry arthroscopy" can be an effective intraoperative technique in the excision of chondroblastomas due to the improved visualization and decreased invasiveness of the approach when compared to standard local excision and curettage techniques.

\section{Abstract}

Introduction: Chondroblastomas are benign, locally aggressive bone tumors that occur in the epiphysis of young patients, requiring surgical excision. The anatomic locations of these lesions pose technical challenges to the surgeon; their proximity to the articular surface and the physis make surgical approach and visualization difficult. This case series describes the use of intramedullary arthroscopic assistance during excisional curettage of three distal femoral chondroblastomas.

Case Report: Three patients with distal femoral chondroblastomas underwent excisional curettage with intraosseous "dry" arthroscopic assistance. We describe a two-tunnel technique, using medial and lateral windows so the arthroscope and working instruments could be within the lesion simultaneously; as well as two variations of a single-tunnel technique.

Conclusion: Achieving adequate exposure and visualization, while protecting the articular surface and physis, during excision of chondroblastomas, is essential for avoiding local recurrence and complications. "Dry" arthroscopic assistance provides the surgeon with an enhanced view of the tumor and tumor cavity, minimizes invasiveness, and has the potential to reduce complications without compromising the completeness of the excision.

Keywords: Chondroblastoma, orthopedic oncology, arthroscopy, dry arthroscopy, bone tumor.

\section{Introduction}

Chondroblastomas are benign, locally aggressive bone tumors that occur within the epiphysis of children. They comprise approximately $1-2 \%$ of primary bone tumors and are painful, progressive lesions, necessitating surgical excision to prevent functional and growth impairments $[1$, $2]$.

Excisions can be complicated by the anatomic location of the tumor: the surgeon must achieve complete excision, while minimizing disruption of the physis and articular surface [3]. Failure to achieve complete excision contributes to the high local recurrence rate of chondroblastoma, and injury to the articular surface may necessitate cartilage restoration or arthroplasty at a young age $[2,3,4]$. Many patients see physeal involvement, which can cause leg length discrepancy or joint alignment issues [4].

Surgical techniques utilizing fluoroscopy to guide curettage have several limitations. Limited cavity visualization requires reliance on the appearance of excised tissue and fluoroscopy to assess the thoroughness of curettage. In some cases, the approach requires an arthrotomy, directly violating the articular surface; others can involve the physis. Considering these limitations, we describe three cases of chondroblastoma of the distal femur in which we performed arthroscopically assisted excision through a transosseous tunnel with direct visualization of the tumor cavity without

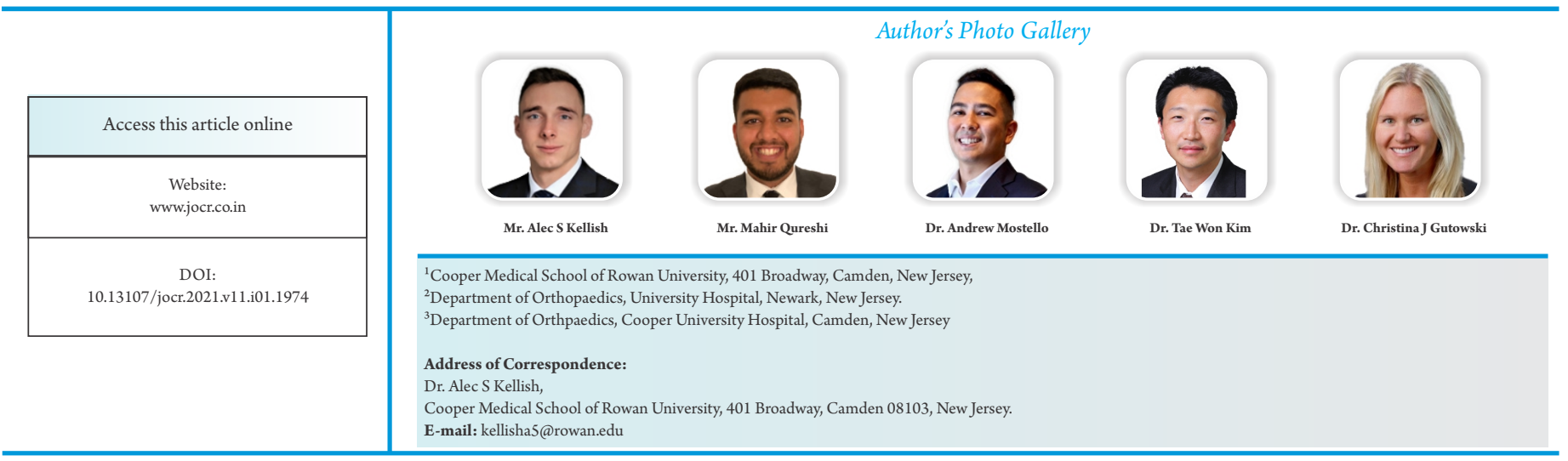

Journal of Orthopaedic Case Reports | pISSN 2250-0685 | eISSN 2321-3817 | Available on www.jocr.co.in | doi:10.13107/jocr.2021.v11.i01.1974 This is an Open Access article distributed under the terms of the Creative Commons Attribution Non-Commercial License (http://creativecommons.org/licenses/by-nc/3.0) which permits unrestricted non-commercial use, distribution, and reproduction in any medium, provided the original work is properly cited. 


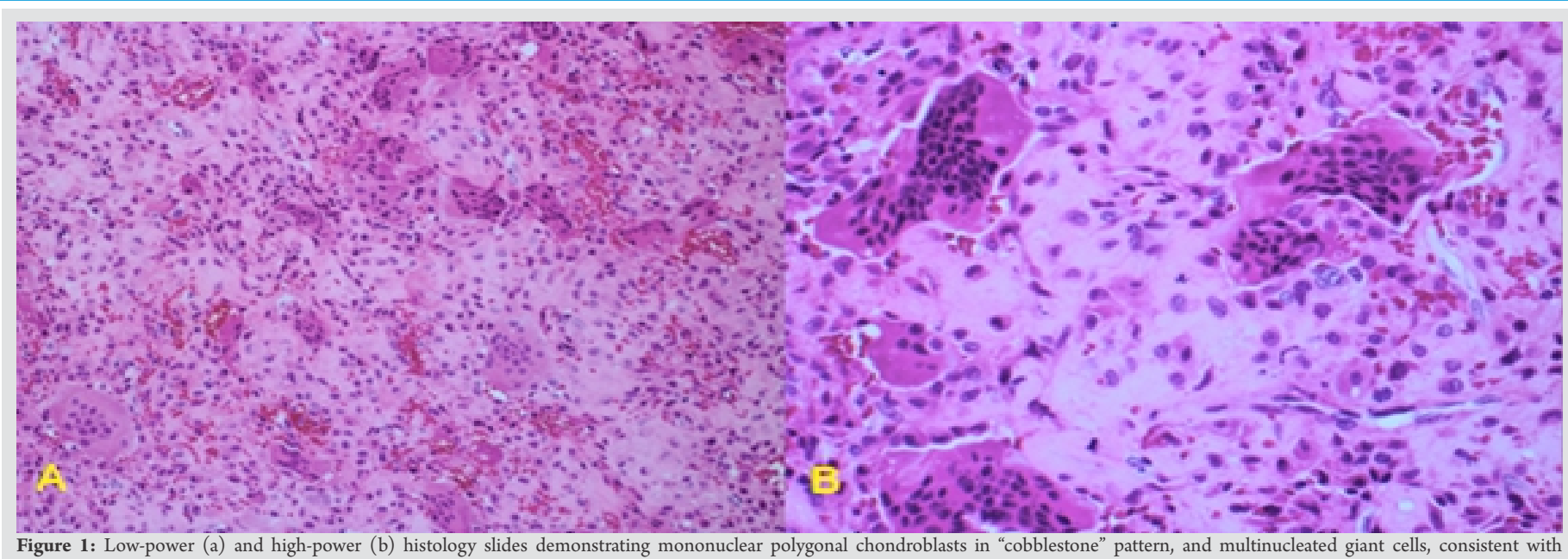

chondroblastoma.

arthrotomy or articular surface violation.

\section{Case Report}

\section{Description of technique}

Orthogonal fluoroscopy is used prior to the incision to localize the tumor within the distal femoral epiphysis. The leg is exsanguinated, and a proximal thigh tourniquet is inflated. A medial or lateral approach to the distal femoral epiphysis is performed through a $2-4 \mathrm{~cm}$ longitudinal incision. A K-wire is then drilled into the lesion, utilizing orthogonal fluoroscopy to confirm the wire's trajectory and depth in the anteroposterior and lateral planes. A $4 \mathrm{~mm}$ diameter trephine reamer is cannulated over the K-wire and the intraosseous tunnel is created.

Once the sclerotic border of the lesion is breached, a $30^{\circ}$ arthroscope without fluid ("dry") is inserted into the tumor cavity through the intraosseous tunnel for intralesional visualization. Arthroscopic instruments including curettes, biters, and pituitary rongeurs are utilized to excise the tumor through the primary tunnel once the arthroscope is removed. The surgeon can decide to create a secondary intraosseous portal to observe the curettage maneuvers being performed from a second angle; this dual-portal technique was employed in Case 2 described below. The primary tunnel can be widened to facilitate observation through a single portal, as described in Case 1. Occasional irrigation is utilized during this "real-time" visualization.

At the conclusion of the curettage, the 30-degree scope is reinserted to examine the cavity and confirm all areas within the cavity have undergone adequate excisional curettage. A 70-degree scope can also follow, to provide additional viewing angles. Adjuvant treatment to the walls of the cavity can be performed at this time, as described in Case 1, when arthroscopic visualization was able to verify treatment of all cavity walls with the high-speed burr.

The defect is then filled with bone graft, fat graft, polymethylmethacrylate, or bone substitute. Arthroscopy can be utilized during the filling step to monitor progression of filling.

\section{Case 1}

A 15-year-old male presented to clinic complaining of 3 months of worsening right knee pain. Imaging revealed a bone lesion within the medial femoral condyle, and open biopsy revealed chondroblastoma (Fig. 1).

Arthroscopically assisted tumor excision was approached

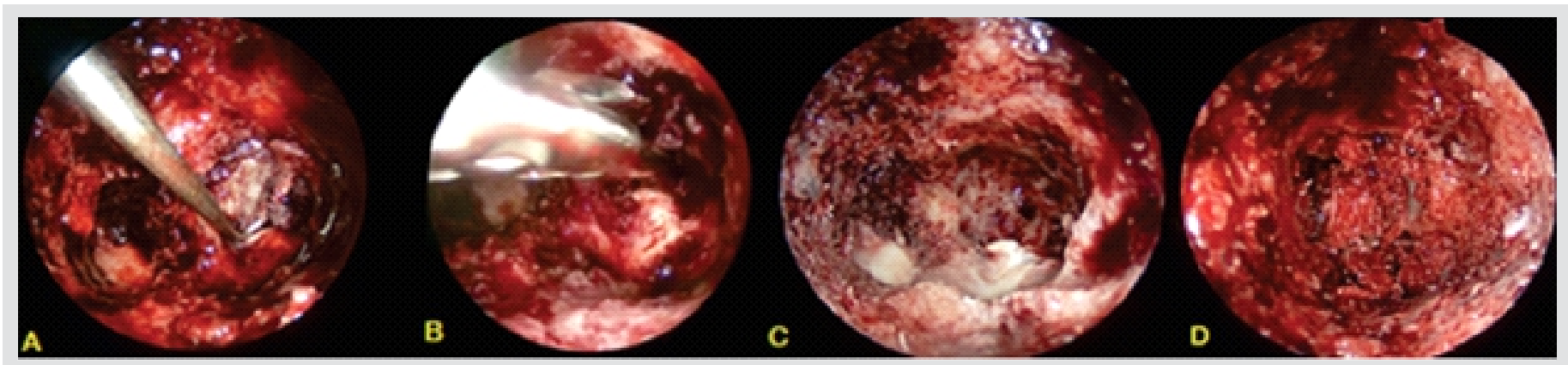

Figure 2: Arthroscopic images of working curette (A), working biter (B), tumor cavity after excision (C), and tumor cavity after grafting (D). These images were obtained via the single tunnel technique. 


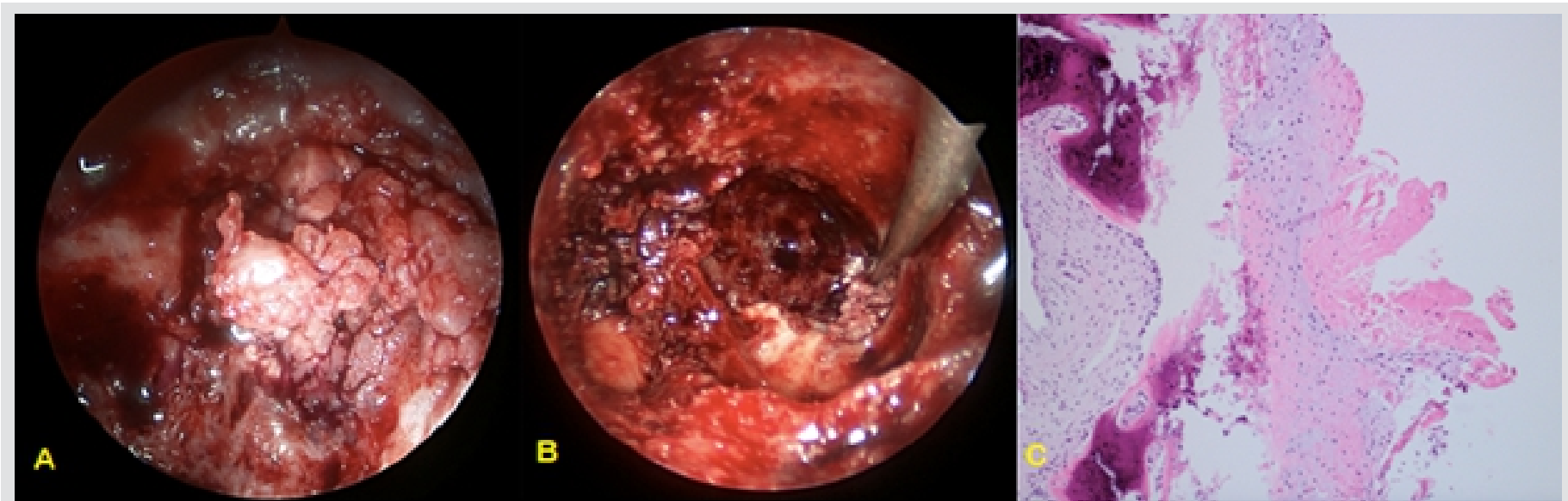

Figure 3: (A and B) Intraoperative arthroscopic image of PCL origin. (C) Histological confirmation of ligamentous tissue biopsied from site.

through a single, widened intraosseous tunnel, accommodating insertion of the arthroscope and instruments simultaneously (Fig. 2). A long burr tip was utilized as adjuvant treatment to the walls of the cavity. With arthroscopic visualization of the tumor cavity, the surgeon was able to confirm complete and thorough treatment of all areas with the burr. Interestingly, the posterior cruciate ligament (PCL) origin was able to be visualized from within the cavity and confirmed histologically (Fig. 3). By utilizing arthroscopic assistance, we were able to avoid disrupting the PCL origin. The residual bony defect and tunnel were then filled with a combination of the bone autograft harvested at the beginning of the case and $10 \mathrm{~mL}$ of ETEX calcium phosphate (Zimmer Biomet, Warsaw IN). This patient is 43 months from surgery with no evidence of local recurrence or

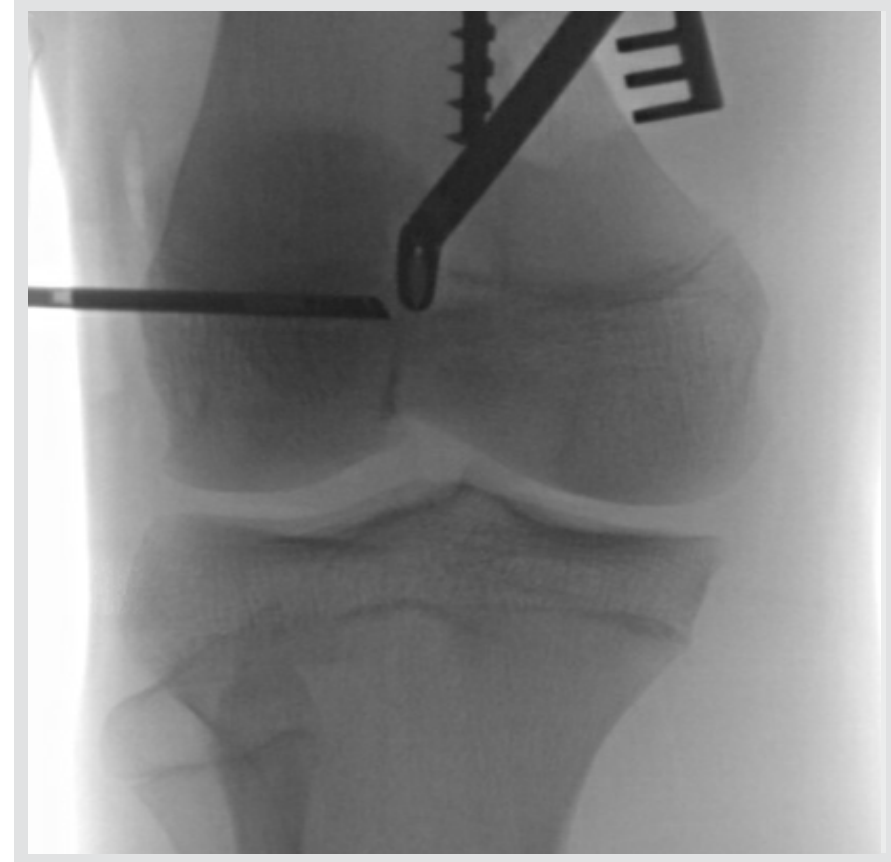

Figure 4: Fluoroscopic image of two-tunnel technique being employed to curette lesion under arthroscopic assistance. knee symptoms.

\section{Case 2}

A 14-year-old male high school basketball player presented complaining of 2 weeks of right knee pain that began after mild trauma. Imaging revealed a lytic lesion within and crossing the distal femoral epiphysis, below the articular surface of the medial trochlea. Needle biopsy confirmed diagnosis of chondroblastoma. Full-length lower extremity $\mathrm{X}$-ray revealed a leg length discrepancy of $4 \mathrm{~mm}$, right longer than left. He underwent excisional curettage of the lesion with arthroscopic assistance, followed by fat grafting in attempt to prevent physeal bar formation. Surgery proceeded according to the two-portal technique, as described above (Fig. 4). The laterally based physeal tunnel

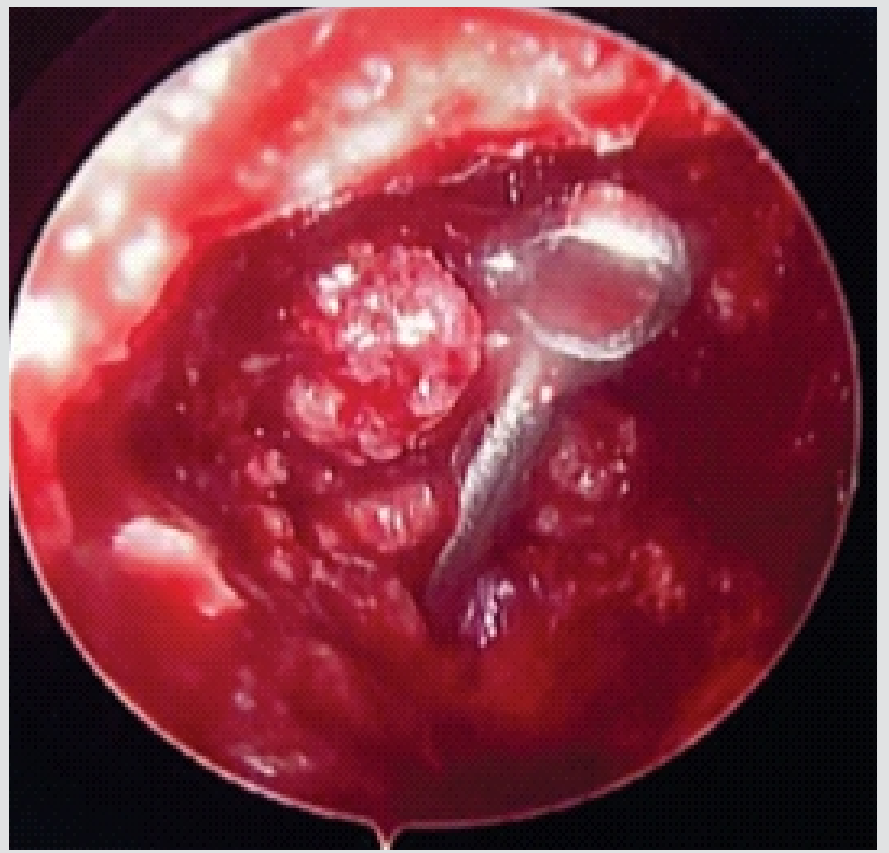

Figure 5: Arthroscopicimage of curettage, performed via two-tunnel technique. 


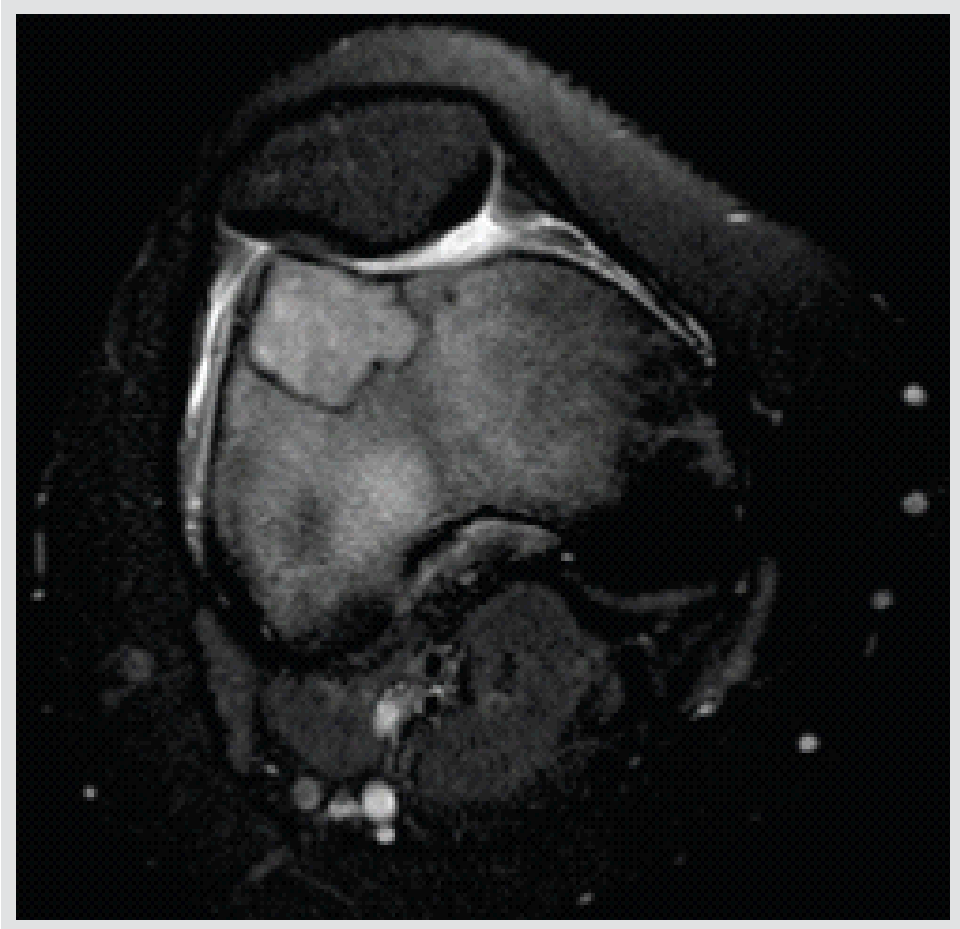

Figure 6: Periarticular chondroblastoma of the right distal femur in a 15-year-old female.

was created distal to the physis, which served as the viewing portal. The working portal was a medially based tunnel, through which instruments were inserted. This two-tunnel technique provided minimal disruption to the physis, while facilitating "real-time" visualization of the excision (Fig. 5). After curettage, fat autograft was inserted into the defect to prevent physeal bar formation. The patient is 12 months from surgery with normal range of motion and function and no evidence of local recurrence.

\section{Case 3}

A 15-year-old female presented to clinic complaining of 4 weeks of worsening atraumatic right knee pain. X-ray and magnetic resonance imaging revealed a marrow replacing $\mathrm{T} 2$ hyperintense lesion abutting the articular cartilage of the lateral trochlea (Fig. 6). Needle biopsy confirmed diagnosis of chondroblastoma.

Due to the distal and lateral nature of this tumor, a singleportal technique was utilized (Fig. 7). The arthroscope and working instruments were inserted into the cavity sequentially, instead of simultaneously. Intraosseous visualization was particularly valuable in this case, because the tumor was extremely close to the articular surface. Cerament, a bone substitute (Bone Support, Lund Sweden) with hydroxyapatite, was utilized to provide immediate structural support to the articular surface without risking thermal injury to the cartilage. The camera reliably

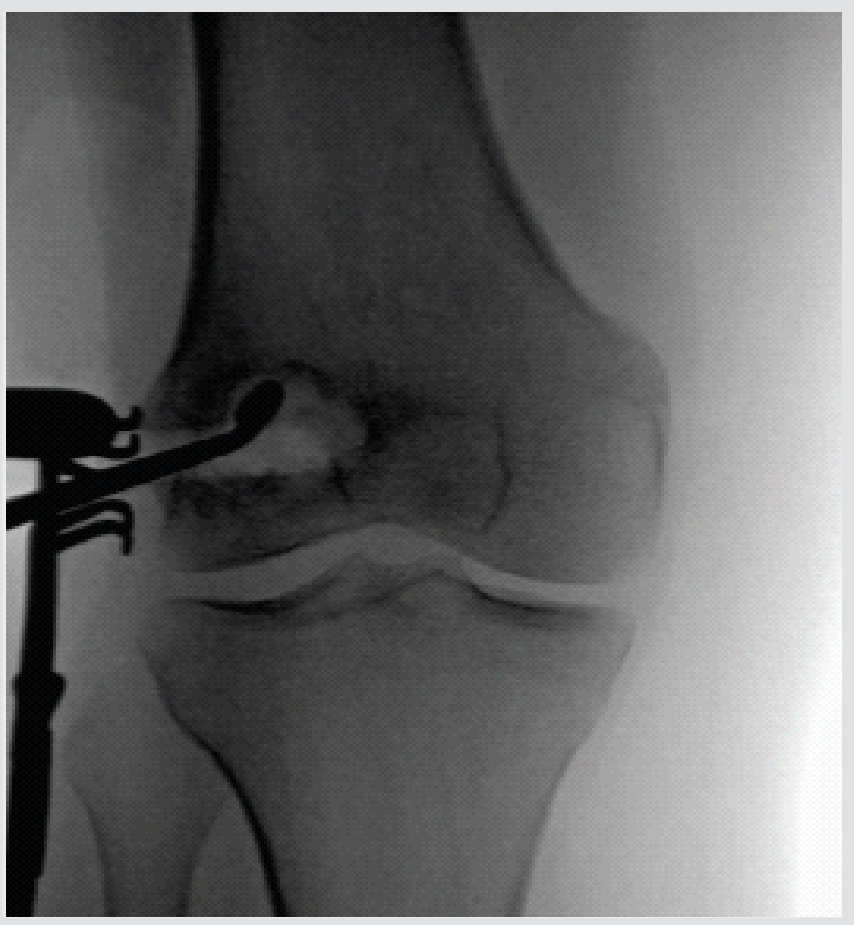

Figure 7: Fluoroscopic image of curettage performed through lateral tunnel

confirmed the cartilage's integrity after curettage. This facilitated safer injection of the bone substitute without extravasation into the knee joint. The patient is 7 months from surgery, with normal range of motion and function, and no evidence of local recurrence.

\section{Discussion}

Excision of epiphyseal tumors of long bones can be challenging due to the anatomical proximity of the physis and articular cartilage. Local recurrence is a common postoperative complication in cases of chondroblastoma and may stem from poor visualization resulting in incomplete tumor excision with traditional fluoroscopy-based technique [3]. The use of an intraosseous arthroscope during chondroblastoma excision can improve intralesional visualization, confirm complete tumor excision, and assess cartilage integrity post-excision. Adjuvant treatments can lower local recurrence rates, but should ideally be utilized under direct visualization $[5,6,7,8,9]$ and are made safer by arthroscopic assistance.

First described in 2018 for treating benign fibrous histiocytomas, an intraosseous, "dry" arthroscopic approach addresses the shortcoming of excisions under fluoroscopic localization methods [10]. Shimizu et al. recommended arthroscopy be limited to patients with small tumors arising within the lateral distal femoral epiphysis, that is, under $1 \mathrm{~cm}$ in diameter, lack epiphyseal plate involvement, and are not a 
chondroblastoma [10]. According to these recommendations, the "dry scope" technique would be contraindicated in all three of our cases. Our work broadens the indications of this technique and demonstrates its role in tumors of various histologies, of various sizes, located in various anatomic locations. Both 1- and 2-portal techniques are described, which can be employed at the surgeon's discretion depending on the anatomic location of the tumor, the integrity of the physis and articular surface, and the necessary angle of visualization.

Arthroscopic assistance for the treatment of epiphyseal bone lesions is not without limitations and/or contraindications. Any malignant bone tumor should be treated with wide en bloc excision, and the use of an intraosseous arthroscope to assist in intralesional excision would be against orthopedic oncologic principles. In addition, a lesion such as an aneurysmal bone cyst or intraosseous hemangioma may continuously bleed during an intralesional excision, making arthroscopic visualization challenging.

\section{Conclusion}

Adequate intralesional visualization during excision of chondroblastomas and other epiphyseal bone tumors is essential for avoiding local recurrence and complications. "Dry" intraosseous arthroscopic assistance addresses the pitfalls of standard techniques and facilitates tumor and tumor cavity visualization. It is safe, minimally invasive, and has the potential to reduce complications without compromising on the completeness of the excision.

\section{Clinical Message}

"Dry arthroscopy" in the excisional curettage of chondroblastomas can be performed effectively and safely in patients with chondroblastomas in which the procedure was previously considered to be contraindicated.

\section{References}

1. Limaiem F, Rawla P. Cancer, chondroblastoma. In: Stat Pearls. Treasure Island, FL: Stat Pearls Publishing; 2020.

2. Xu H, Nugent D, Monforte HL, Binitie OT, Ding Y, Letson GD, et al. Chondroblastoma of bone in the extremities: A multicenter retrospective study. J Bone Joint Surg Am 2015;97:925-31.

3. Özer D, Arıkan Y, Gür V, Gök C, Akman YE. Chondroblastoma: An evaluation of the recurrences and functional outcomes following treatment. Acta Orthop Traumatol Turc 2018;52:415-8.

4. Farfalli GL, Slullitel PA, Muscolo DL, Ayerza MA, AponteTinao LA. What happens to the articular surface after curettage for epiphyseal chondroblastoma? A report on functional results, arthritis, and arthroplasty. Clin Orthop Relat Res 2017;475:760-6.

5. Schreuder HW, Pruszczynski M, Veth RP, Lemmens JA. Treatment of benign and low-grade malignant intramedullary chondroid tumours with curettage and cryosurgery. EurJ Surg Oncol 1998;24:120-6.

6. DiCaprio M, Boyle J, Gibbs C. Use of the argon beam coagulator as an adjuvant for treating bone tumors. Tech Orthop 2007;22:110-5.

7. Outani H, Kakunaga S, Hamada K, Takenaka S, Nakai S, Yasuda N, et al. Clinical outcomes of chondroblastoma treated using synthetic bone substitute: Risk factors for developing radiographic joint degeneration. World J Surg Oncol2020;18:47.

8. van der Geest IC, de Valk MH, de RooyJW, Pruszczynski M, Veth RP, Schreuder HW. Oncological and functional results of cryosurgical therapy of enchondromas and chondrosarcomas grade 1.J Surg Oncol 2008;98:421-6.

9. Lewis VO, Wei A, Mendoza T, Primus F, Peabody T, Simon MA. Argon beam coagulation as an adjuvant for local control of giant cell tumor. Clin Orthop Relat Res 2007;454:192-7.

10. Shimizu J, Emori M, Okada Y, Hasegawa T, Yamashita T. Arthroscopic resection for benign fibrous histiocytoma in the epiphysis of the femur. Case Rep Orthop 2018;2018:8030862.
Conflict of Interest: Nil Source of Support: Nil

Consent: The authors confirm that informed consent was obtained from the patient for publication of this case report

\section{How to Cite this Article}

Kellish AS, Qureshi M, Mostello A, Kim TW, Gutowski CJ. "Dry Arthroscopy" is a Valuable Tool in the Excisional Curettage of Chondroblastoma: A Case Series. Journal of Orthopaedic Case Reports 2021 January; 11(1): 82-86. 